\title{
EFECTO IN VITRO DEL EXTRACTO ETANÓLICO DE CAULERPA FILIFORMIS EN PARÁMETROS SEMINALES HUMANOS
}

\author{
Egg, K. \\ Avia, S. \\ Villalobos, L. \\ Wong, Y. \\ Gonzales Figueroa, H. ${ }^{1}$
}

\section{RESUMEN}

El objetivo de este estudio fue determinar el efecto in vitro del extracto etanólico de Caulerpa filiformis en parámetros seminales humanos. Se realizaron las siguientes pruebas: vitalidad, motilidad y dispersión de cromatina espermática; tomándose en cuenta las variables tiempo $\left(5^{\prime}, 15^{\prime}, 35^{\prime}\right.$ y $\left.60^{\prime}\right)$ y concentración $(0.04 \mathrm{mg} / \mathrm{ml}$, $0.4 \mathrm{mg} / \mathrm{ml} \mathrm{y} 4 \mathrm{mg} / \mathrm{ml}$ ) para cada prueba. La muestra fue diluida hasta una concentración de $10 \times 10^{6}$ de espermatozoides $/ \mathrm{ml}$ con suero fisiológico al $0.9 \%$, luego incubada $1: 1(\mathrm{v} / \mathrm{v})$ con el extracto. Con respecto a la vitalidad, a partir de los 15 minutos el extracto produjo mortalidad espermática. La $\mathrm{DL}_{50}$ fue de $17.35 \mathrm{mg} / \mathrm{ml}(11.08-57.17$ $\mathrm{mg} / \mathrm{ml}), 8.75 \mathrm{mg} / \mathrm{ml}(7.15-12.00 \mathrm{mg} / \mathrm{ml})$ y $6.43 \mathrm{mg} / \mathrm{ml}(5.53-8.02$ $\mathrm{mg} / \mathrm{ml}$ ) para los $15^{\prime}, 35^{\prime}$ y 60 ', respectivamente. En cuanto a la motilidad espermática se observó una disminución significativa con respecto al control en la concentración de $4 \mathrm{mg} / \mathrm{ml}$ en los $15^{\prime}$ y $35^{\prime}$. A los 60' en dicha concentración, todas las repeticiones alcanzaron una inmovilización espermática total. La $\mathrm{CE}_{50}$ a los 60 ' fue de 0.49 $\mathrm{mg} / \mathrm{ml}(-1.25-1.16 \mathrm{mg} / \mathrm{ml})$. La prueba de ANOVA de 2 vías mostró que tanto el porcentaje de mortalidad como de espermatozoides inmovilizados se vio afectado por las variables tiempo y concentración, mientras que la interacción de éstas solo afectó la motilidad. El parámetro motilidad solo muestra un efecto dosis dependiente en la concentración de $4 \mathrm{mg} / \mathrm{ml}$. La fragmentación de ADN no se vio afectada por ninguna de las variables ya mencionadas ni por la interacción de estas. Los resultados obtenidos con el extracto etanólico de Caulerpa filiformis podrían tener una naturaleza contraceptiva

1 Laboratorio de Biotecnología y Fisiología Animal. Facultad de Ciencias Biológicas, Universidad Ricardo Palma. e-mail: karina.egga@urp.pe 
promisoria, y tal vez ser tomada en cuenta como una alternativa a los espermicidas comerciales, para ello se necesitará futuros estudios que develen la composición química de esta especie.

Palabras claves: Caulerpa filiformis, Dispersión de Cromatina Espermática (DCE) parámetros seminales.

\section{SUMMARY}

The goal of this study was to define in vitro effect of ethanol extract of Caulerpa filiformis in human semen parameters. The following tests were performed: vitality, motility and sperm chromatin dispersion; taking into account the time variable $\left(5^{\prime}, 15^{\prime}, 35^{\prime}\right.$ and $\left.60^{\prime}\right)$ and concentration $(0.04 \mathrm{mg} / \mathrm{ml}, 0.4 \mathrm{mg} / \mathrm{ml}$ and $4 \mathrm{mg} / \mathrm{ml})$ for each test. The trial was diluted to a concentration of $10 \times 10^{6}$ sperm $/ \mathrm{ml}$ with $0.9 \%$ saline, then incubated 1: $1(\mathrm{v} / \mathrm{v})$ with the extract. Concerning vitality, from 15 minutes to extract sperm mortality occurred. LD50 was $1735 \mathrm{mg} / \mathrm{ml}$ (11.08-57.17 mg / ml), $8.75 \mathrm{mg} / \mathrm{ml}(7.15-12.00 \mathrm{mg} / \mathrm{ml})$ and $6.43 \mathrm{mg}$ / $\mathrm{ml}(5.53-8.02 \mathrm{mg} / \mathrm{ml})$ for 15', 35' and 60', respectively. As for sperm motility, it was observed a significant decrease relative to the control in the concentration of $4 \mathrm{mg} / \mathrm{ml}$ in 15 'and 35'. LD50 was $1735 \mathrm{mg} / \mathrm{ml}$ $(11.08-57.17 \mathrm{mg} / \mathrm{ml}), 8.75 \mathrm{mg} / \mathrm{ml}(7.15-12.00 \mathrm{mg} / \mathrm{ml})$ and $6.43 \mathrm{mg}$ / $\mathrm{ml}(5.53-8.02 \mathrm{mg} / \mathrm{ml})$ for $15^{\prime}, 35^{\prime}$ and 60', respectively. As for sperm motility significant decrease relative to the control it was observed in the concentration of $4 \mathrm{mg} / \mathrm{ml}$ in 15' and 35'. At 60'in such concentration, all occurrences attain complete sperm immobilization. The EC50 at 60 minutes was $0.49 \mathrm{mg} / \mathrm{ml}(-1.25-1.16 \mathrm{mg} / \mathrm{ml})$.

As for sperm motility significant decrease relative to the control it was detected in the concentration of $4 \mathrm{mg} / \mathrm{ml}$ in 15'and 35'. At 60'in such concentration, all occurrences attain complete sperm immobilization. The EC50 at 60 minutes was $0.49 \mathrm{mg} / \mathrm{ml}(-1.25-1.16 \mathrm{mg} / \mathrm{ml})$. Test 2-way ANOVA showed that both the percentage of mortality and frozen sperm was affected by the variables of time and concentration, while their interaction affected only motility. The motility parameter shows only a dose dependent effect on the concentration of $4 \mathrm{mg} / \mathrm{ml}$. DNA fragmentation was not affected by any of the variables mentioned above or by the interaction of these. The results obtained with the ethanol extract of Caulerpa filiformis could have a promising contraceptive nature, and perhaps be taken into account 
as an alternative to commercial spermicides, for that future studies that reveal the chemical composition of this species need.

Keywords: Caulerpa filiformis, Sperm Chromatin Dispersion (SCD) seminal parameters.

\section{INTRODUCCIÓN}

Los organismos marinos son una fuente incalculable de metabolitos con amplia acción farmacológica, dentro de ellas las algas son uno de los grupos más importantes que habita en este ecosistema y a su vez son fuentes de compuestos químicos de diversas actividades biológicas que merecen ser investigadas (Mayer \& Hamann, 2002).

Las especies que han cobrado importancia en estos últimos años son las del género Caulerpa (Chlorophyta), ya que ha presentado una inusual característica invasiva en otras latitudes como Australia y el mar Mediterráneo debido a los cambios de temperatura de las masas de agua (Cummings \& Williamson, 2008) (Israel, Einav, \& Seckbach, 2010) (Lemée et al., 1993). En el Perú poseemos una especie de este género, la Caulerpa filiformis, la cual está presentando un comportamientos invasivo como las demás especies de su género. Los planes de control están basados en una agresiva erradicación del alga y no han tenido mayor éxito. Ante esta situación de gran aumento de biomasa se propone el uso de esta alga por sus metabolitos presentes (Israel et al., 2010), en estudios asociados a actividades anticancerígenas, anti inflamatorias, anti proliferativa, antimicrobiana, anti herpética y antivirales (Richter et al., 2014)(Cengiz et al., 2012)(Da Matta et al., 2011) (Nicoletti et al., 1999).

Entre los compuestos que destacan en esta alga está el sesquiterpenoide caulerpinina presente en todas las especies del género. Esta molécula apolar predomina frente a los demás metabolitos, alcanzado un $1.3 \%$ del peso seco del alga (Smyrniotopoulos et al., 2003). No obstante, se ha tomado poca atención a las actividades biológicas que presentan extractos polares, los cuales también poseen metabolitos de interés como los polifenoles (flavonoides) (Glombitza and Keusgen, 1995). Asimismo, los extractos polares de Caulerpa filiformis han demostrado repeler a los herbívoros Aplysia sydneyensis y Turbo torquatus, lo que sugiere la presencia de moléculas polares, aún no identificadas, como responsables de dicha actividad (Davis et al., 2005). 
Se ha comprobado en muchas investigaciones el efecto espermicida de extractos vegetales en animales vertebrados e invertebrados de estructura similar a los sesquiterpernos como los aislado de la esponja marina Geodesia que inhibe la motilidad espermática del Tetrapygus niger mediante la supresión de la lanzadera de la fosfoqueratinasa que afecta directamente a la dineína ATPasa (Ohta et al., 2008); el sesquiterpeno aislado del alga Laurencia claviformis presenta una inhibición en la citokinesis de los espermatozoides de Tetrapygus niger hacia los ovocitos (Rovirosa et al., 1999); en Mus musculus se reportó inmovilización espermática en un $100 \%$ así como una considerable disminución de viabilidad con el extracto de Heliopis longipes y Risinus communis (Martinez-Loredo et al., 2015) (Nithya et al., 2012); así como el extracto de Polygala tenuifolia y Stephania hernandifolia-Achyranthes aspera reportaron una inmovilización espermática total y reducción de viabilidad en espermatozoides humanos (Qiu et al., 2011) (Paul et al., 2006).

Sin embargo fueron Prakash et al., 2013 los primeros en reportar sobre la actividad espermicida de algas sobre espermatozoides humanos, generando una inquietud acerca del uso de estos metabolitos en el ámbito de la contraceptividad.

Existe muy poca literatura sobre el impacto de los metabolitos secundarios de las algas sobre la capacidad reproductiva en otros organismos, y discreta información acerca de estos compuestos en las células gonadales humanas como los espermatozoides, por lo tanto investigaciones en ésta área son requeridas.

El presente estudio tuvo como objetivo determinar los efectos del extracto etanólico de Caulerpa filiformis en parámetros seminales en individuos normospérmicos.

\section{METODOLOGÍA}

\section{Colección de algas y obtención de extractos crudos}

Se colectó las algas del sector submareal de la playa Pucusana, Lima, Perú. Las algas colectadas fueron lavadas con agua de mar, posteriormente con agua potable y secada en la estufa a $37^{\circ} \mathrm{C}$ por 12 horas. Posteriormente fueron pesadas, cortadas y trituradas. Para obtener el extracto etanòlico, se desengraso 2.5 gramos de la muestra en $25 \mathrm{ml}$ de cloroformo por 72 horas en oscuridad y agitación constante. Se filtró el macerado en 
papel Whatman $\mathrm{N}^{\mathrm{o}} 1$ y el residuo del extracto fue macerado nuevamente en un volumen igual de cloroformo por 48 horas para asegurar un completo desengrase. El residuo se colocó en $25 \mathrm{ml}$ de etanol absoluto en oscuridad por 72 horas. La muestra se filtró y el extracto etanólico fue llevado a concentración a 40 grados centígrados toda la noche y se resuspendió en suero hasta llegar a una concentración final de $8 \mathrm{mg} / \mathrm{ml}$. El extracto clorofórmico se obtuvo del primer desengrase por 72 horas y el solvente se evaporo a 37 grados durante toda la noche, posteriormente se resuspendió en DMSO y se diluyo con suero hasta obtener una concentración del solvente al 1\% y de extracto, una concentración $0.082 \mathrm{mg} / \mathrm{ml}$. Finalmente los extractos se almacenaron $4^{\circ} \mathrm{C}$ para su posterior uso.

\section{Selección de individuos normospérmicos}

Todos los voluntarios que participaron en esta investigación tuvieron que firmar un consentimiento informado sobre el uso de su eyaculado. Las muestras de semen fueron recolectadas en frascos estériles y obtenidas por masturbación con 3 días de abstinencia sexual y estar en una edad reproductiva entre 20 a 25 años. Se mantuvo en la estufa a $37^{\circ} \mathrm{C}$ por $45 \mathrm{mi}-$ nutos para facilitar su licuefacción. Para determinar la calidad espermática de los individuos sólo se aceptaron muestras con una concentración mayor o igual a 15 millones de espermatozoides $/ \mathrm{ml}$; mayores o iguales a $40 \%$ de espermatozoides progresivos para motilidad; mayores e iguales a $58 \%$ para vitalidad.

Determinación del efecto in vitro de los extractos crudos de Caulerpa filiformis Para la determinación del efecto in vitro de los extractos crudos en parámetros seminales humanos, se tomaron los valores de la motilidad, fragmentación de ADN y vitalidad en tiempos de incubación de $5,15,35$ y 60 minutos. Cada muestra fue diluida hasta una concentración de 10 millones de espermatozoides por mililitro con suero, luego fue incubada $1: 1(\mathrm{v} / \mathrm{v})$ con el extracto etanólico formando las siguientes concentraciones: $4 \mathrm{mg} / \mathrm{ml}, 0.4 \mathrm{mg} / \mathrm{ml}, 0.04 \mathrm{mg} / \mathrm{ml}$ y un control(suero fisiológico). En caso del extracto clorofórmico las concentraciones fueron las siguientes $41 \mathrm{ug} / \mathrm{ml}, 4.1 \mathrm{ug} / \mathrm{ml}, 0.41 \mathrm{ug} / \mathrm{ml}$ y un control de DMSO 0.5\%.

\section{Prueba de motilidad}

Se usó $10 \mu 1$ de la muestra incubada y se cargó en la cámara de Neubauer grabándose 3 campos por un lapso 2 segundos por cada uno. Se consideró inmótiles a los espermatozoides que presentaba una motilidad nula y 
además a los que tenían una motilidad in situ de bajo movimiento flagelar.

\section{Prueba de Dispersión de Cromatina Espermática (DCE)}

Se utilizó $30 \mu 1$ de dilución, y se mezcló con $70 \mu 1$ de agarosa semisólida de bajo punto de fusión al 1\%. Esta suspensión se colocó en portaobjetos pre tratados con agarosa solidificada al $0.65 \%$ y se cubrió cuidadosamente con el cubreobjetos hasta la solidificación de la mezcla a $4^{\circ} \mathrm{C}$ para luego retirar el cubreobjeto. La lámina se incubara por 7 minutos en una solución ácida de denaturación a oscuridad provista por el fabricante para luego incubarla en la solución de neutralización por 10 minutos a temperatura ambiente. Las muestras serán lavadas en Buffer de lavado por 25 minutos que luego fueron deshidratadas en baños secuenciales de $70 \%$, 90\% y $96 \%$ de Etanol de dos minutos respectivamente. Las láminas fueron teñidas con tinción DiffQuik según las instrucciones del fabricante. Los espermatozoides de halos pequeños y los que no presentaron halos fueron considerados espermatozoides fragmentados y los de halos grandes, espermatozoides no fragmentados.

\section{Prueba de vitalidad}

Para determinar la vitalidad se usó una alícuota de $10 \mu 1$ de muestra y se mezcló con $10 \mu \mathrm{l}$ de Eosina-nigrosina, luego se colocó $10 \mu \mathrm{l}$ de dicha mezcla en un portaobjeto y se procedió a hacer el frotis. Los espermatozoides no coloreados y ligeramente rosas se consideraron vivos y los coloreados rojos intensamente muertos.

Figura 1.

Valores porcentuales del parámetro vitalidad y motilidad con respecto al tiempo de exposición al extracto etanólico de Caulerpa filiformis. (a) \% de espermatozoides muertos en las diferentes concentraciones del extracto a cuatro tiempos diferentes. (b) \% de espermatozoides inmóviles en las diferentes concentraciones del extracto a cuatro tiempos diferentes.
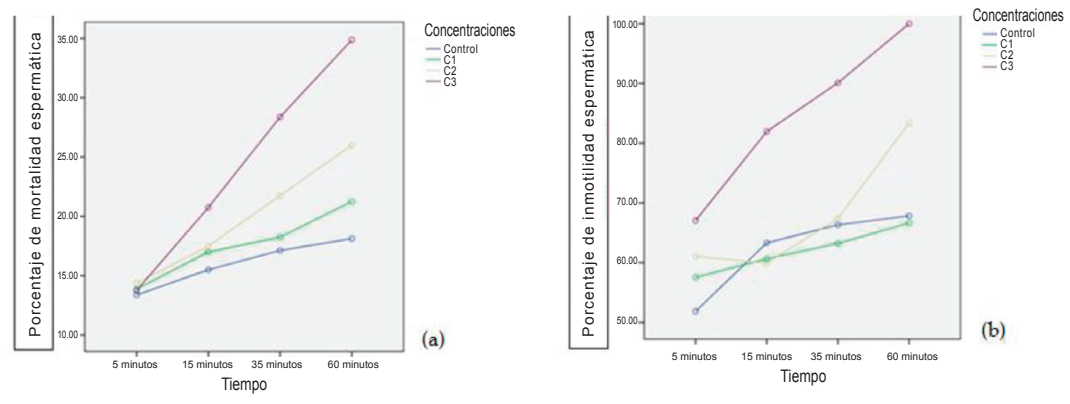


\section{Análisis estadístico}

La normalidad y homocedasticidad de los datos se evaluaron con las pruebas de Shapiro-Wilk y Levene, respectivamente. Para determinar la concentración máxima a la cual no se observa efecto (NOEC) y la mínima a la cual se observa efecto (LOEC) para cada tiempo, en relación al porcentaje de espermatozoides no fragmentados, de mortalidad y porcentaje de inmovilidad espermática, se aplicó la prueba de ANOVA de 1 vía con el post-hoc de Dunnet. En caso no se cumplieran los supuestos, la prueba no paramétrica de Kruskal-Wallis fue llevada a cabo utilizando la prueba de U de Mann-Whitney como prueba post-hoc. Para evaluar el porcentaje de mortalidad e inmovilización de espermatozoides, se calculó la $\mathrm{DL}_{50} \mathrm{y}$ $\mathrm{CE}_{50}$, respectivamente, para cada tiempo mediante el método de regresión Probit. Con el fin de evaluar el efecto de la variable tiempo y concentración, así como su interacción, en los parámetros seminales previamente mencionados se utilizó la prueba de ANOVA de 2 vías. Todos los análisis se realizaron con un $95 \%$ de confiabilidad en el paquete estadístico SPSS v.21.

\section{RESULTADOS}

La prueba de DCE no presentó diferencia significativa entre los tratamientos en relación al porcentaje de espermatozoides no fragmentados en todos los tiempos ( $\mathrm{p}>0.05)$ (Tabla 1), lo cual sugiere la ausencia de una actividad genotóxica. Con respecto al porcentaje de mortalidad espermática, se produjo un efecto a partir de los 15 minutos. El NOEC y LOEC para dicho parámetro a los $15^{\prime}$ y $35^{\prime}$ fue de $0.4 \mathrm{mg} / \mathrm{ml}$ y $4 \mathrm{mg} / \mathrm{ml}$, respectivamente. A los 60' la NOEC fue de $0.04 \mathrm{mg} / \mathrm{ml}$ y la LOEC $0.4 \mathrm{mg} / \mathrm{ml}$. La $\mathrm{DL}_{50}$ fue de $17.35 \mathrm{mg} / \mathrm{ml}(11.08-57.17 \mathrm{mg} / \mathrm{ml}), 8.75 \mathrm{mg} / \mathrm{ml}(7.15-$ $12.00 \mathrm{mg} / \mathrm{ml}), 6.43 \mathrm{mg} / \mathrm{ml}(5.53-8.02 \mathrm{mg} / \mathrm{ml})$ para los $15^{\prime}, 35^{\prime}$ у 60 ', respectivamente. demostrando así que no existe una actividad citotóxica. Con respecto al porcentaje de espermatozoides inmovilizados, tan solo se observó un aumento significativo con respecto al control en la concentración de $4 \mathrm{mg} / \mathrm{ml}$ en los $15^{\prime}$ y $35^{\prime}$. A los $60^{\prime}$ en dicha concentración, todas las repeticiones alcanzaron $100 \%$ de espermatozoides inmovilizados 
Tabla 1. Porcentaje (\%) de espermatozoides no fragmentados, $\%$ de mortalidad y $\%$ de espermatozoides inmovilizados en extracto etanólico de Caulerpa filiformis de $4 \mathrm{mg} / \mathrm{ml}, 0.4 \mathrm{mg} / \mathrm{ml}$ y $0.04 \mathrm{mg} / \mathrm{ml}$

\begin{tabular}{|c|c|c|c|c|c|c|c|c|c|c|c|c|c|c|c|c|}
\hline & \multicolumn{4}{|c|}{ Control } & \multicolumn{4}{|c|}{$0.04 \mathrm{mg} / \mathrm{ml}$} & \multicolumn{4}{|c|}{$0.4 \mathrm{mg} / \mathrm{ml}$} & \multicolumn{4}{|c|}{$4 \mathrm{mg} / \mathrm{ml}$} \\
\hline & 5 & $15^{\circ}$ & $35^{\circ}$ & $60^{\circ}$ & 5 & $15^{\prime}$ & $35^{\circ}$ & $60^{\circ}$ & 5 & $15^{\prime}$ & $35^{\circ}$ & $60^{\prime}$ & 5 & $15^{\prime}$ & $35^{\circ}$ & $60^{\circ}$ \\
\hline 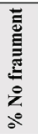 & $\begin{array}{l}5.25 \\
\pm 2 . \\
82\end{array}$ & \begin{tabular}{|c|}
$7.00 \pm$ \\
6.93
\end{tabular} & $\begin{array}{c}4.50 \pm \\
2.56\end{array}$ & $\begin{array}{c}3.63 \pm \\
1.51\end{array}$ & \begin{tabular}{|c|}
$4.87 \pm$ \\
3.14
\end{tabular} & $\begin{array}{c}6.25 \pm \\
4 . \mathrm{S} 9\end{array}$ & \begin{tabular}{|c|}
$4.50 \pm$ \\
2.45
\end{tabular} & \begin{tabular}{|c|}
$4 . \mathrm{SS}$ \\
\pm 2.1 \\
0
\end{tabular} & $\begin{array}{c}5.86 \pm \\
3.90\end{array}$ & $\begin{array}{c}6.00 \pm \\
4.53\end{array}$ & \begin{tabular}{|c|}
5.75 \\
\pm 2.4 \\
9
\end{tabular} & \begin{tabular}{|c|}
$3.63 \pm$ \\
1.85
\end{tabular} & $\begin{array}{c}6.13 \pm \\
3.60\end{array}$ & $\begin{array}{c}7.00 \pm \\
3.59\end{array}$ & $\begin{array}{c}7.00 \pm 5 \\
60\end{array}$ & $\begin{array}{c}\text { 4.SS } \pm 3 . \\
31\end{array}$ \\
\hline 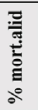 & $\begin{array}{c}13.3 \\
\mathrm{~S} \pm 7 \\
.09\end{array}$ & $\begin{array}{l}15.50 \\
\pm 3.78\end{array}$ & $\begin{array}{l}17.13 \\
\pm 4.26\end{array}$ & $\begin{array}{l}18.13 \\
\pm 2.36\end{array}$ & \begin{tabular}{|l|}
13.58 \\
\pm 4.73
\end{tabular} & $\begin{array}{r}17.00 \\
+1.85\end{array}$ & $\begin{array}{l}18.25 \\
\pm 4.30\end{array}$ & \begin{tabular}{|l|}
21.25 \\
\pm 4.9 \\
$\mathrm{~S}$
\end{tabular} & $\begin{array}{l}14.3 \mathrm{~S} \\
\pm 5.40\end{array}$ & $\begin{array}{r}17.50 \\
\pm 4.14\end{array}$ & \begin{tabular}{|c|}
21.75 \\
\pm 4.7 \\
1
\end{tabular} & $\begin{array}{c}26.00 \\
\pm 1.69 \\
*\end{array}$ & $\begin{array}{l}13.75 \\
\pm 4.59\end{array}$ & $\begin{array}{c}20.75 \\
\pm 2.19 \\
*\end{array}$ & \begin{tabular}{|c|}
$28.38 \pm$ \\
$3.74^{*}$
\end{tabular} & \begin{tabular}{|c|}
$34.8 \mathrm{~S} \pm$ \\
$8.36^{*}$
\end{tabular} \\
\hline 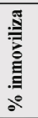 & $\begin{array}{l}51.8 \\
6 \pm 1 \\
3.12\end{array}$ & \begin{tabular}{|l|}
63.31 \\
\pm 11.4 \\
5
\end{tabular} & $\begin{array}{l}66.34 \\
\pm 14.2 \\
3\end{array}$ & $\begin{array}{l}67.83 \\
\pm 19.6 \\
7\end{array}$ & \begin{tabular}{|l|}
57.54 \\
\pm 13.6 \\
1
\end{tabular} & $\begin{array}{l}60.64 \\
\pm 13.5 \\
\mathrm{~S}\end{array}$ & $\begin{array}{l}63.23 \\
\pm 12.6 \\
2\end{array}$ & \begin{tabular}{|l|}
66.65 \\
\pm 8.0 \\
0
\end{tabular} & $\begin{array}{l}61.05 \\
\pm 15.1 \\
2\end{array}$ & $\begin{array}{l}59.91 \\
\pm 12.6 \\
4\end{array}$ & \begin{tabular}{|l|}
67.40 \\
\pm 9.5 \\
2
\end{tabular} & \begin{tabular}{|l|}
83.37 \\
\pm 14.8 \\
0
\end{tabular} & $\begin{array}{l}67.05 \\
\pm 14.7 \\
9\end{array}$ & $\begin{array}{l}81.95 \\
\pm 6.63 \\
*\end{array}$ & \begin{tabular}{|l}
$90.11 \pm$ \\
$11.81^{*}$
\end{tabular} & $\begin{array}{l}100+0 . \\
00-\end{array}$ \\
\hline
\end{tabular}

$\mathrm{La} \mathrm{CE}_{50}$ a los 60' fue de $0.49 \mathrm{mg} / \mathrm{ml}(-1.25-1.16 \mathrm{mg} / \mathrm{ml})$. La prueba de ANOVA de 2 vías mostró que el porcentaje de mortalidad e inmotilidad espermática se vio afectado por los factores "Tiempo" y "Concentración". Solo existió diferencia significativa en la interacción entre estos factores

Tabla 2.

Prueba de ANOVA de 2 vías para las variables "Tiempo" y "Concentración" en los parámetros $\%$ mortalidad y $\%$ de espermatozoides inmovilizados.

\begin{tabular}{|c|c|c|c|c}
\hline Parámetros seminales & Variable & F & gl & Sig. \\
\hline \multirow{3}{*}{$\%$ de mortalidad } & Tiempo & 34.88 & 3 & $<0.001$ \\
\cline { 2 - 5 } & Concentración & 20.11 & 3 & $<0.001$ \\
\cline { 2 - 5 } & Interacción & 3.80 & 9 & $<0.001$ \\
\hline \multirow{2}{*}{$\begin{array}{c}\text { de espermatozoides } \\
\text { inmovilizados }\end{array}$} & Tiempo & 10.69 & 3 & $<0.001$ \\
\cline { 2 - 5 } & Concentración & 16.98 & 3 & $<0.001$ \\
\cline { 2 - 5 } & Interacción & 1.12 & 9 & $\mathbf{0 . 3 6 2}$ \\
\hline
\end{tabular}

\section{DISCUSIÓN}

La vitalidad, motilidad y recientemente la fragmentación de ADN en espermatozoides son factores importantes para la correcta función de los mismos. La evaluación de la vitalidad es uno de los elementos básicos 
del análisis seminal, y es especialmente importante en las muestras donde muchos espermatozoides son inmóviles, para distinguir entre espermatozoides inmóviles muertos y espermatozoides inmóviles vivos. La tinción eosina-nigrosina puede proporcionar un análisis complementario a la evaluación de la motilidad, ya que el porcentaje de las células muertas no debe exceder el porcentaje de espermatozoides inmóviles (WHO 2010). La fragmentación del ADN espermático está siendo reconocida cada vez más como una prueba indicadora de infertilidad. Gandini et al. (2000) informaron que la presencia de la fragmentación del ADN en espermatozoides eyaculados podría correlacionar con defectos en la espermatogénesis. Por esta razón incluimos la prueba de Dispersión de Cromatina Espermática (DCE) como un método de determinación de la fragmentación de ADN espermático, basándonos en el principio de que los espermatozoides fragmentados no producen el halo característico por la dispersión de los bucles de ADN.

La prueba DCE realizada en la presente investigación no presentó diferencia significativa entre las concentraciones y el control en ningún rango de tiempo ( $\mathrm{p}>0.05)$, mostrando un índice de fragmentación del $5 \%$ en el máximo tiempo de exposición a $4 \mathrm{mg} / \mathrm{ml}$. lo cual nos indica que la exposición a la fracción etanólica no produce daño a la cromatina espermática, por lo tanto se podría aseverar que no presenta una actividad genotóxica, coincidiendo así con los resultados obtenidos por Paul et al. (2006) lo cual registró un valor de $12 \%$ de fragmentación espermática frente al extracto crudo de Achyranthes aspera y Stephania hernandifolia, a su vez tampoco mostró diferencia significativa con respecto al control (11\%).

Los resultados obtenidos en cuanto a la motilidad mostraron un porcentaje de inmovilización del $100 \%$ en $4 \mathrm{mg} / \mathrm{ml}$ a los 60 ', la $\mathrm{CE}_{50}$ tan solo llego a $0.49 \mathrm{mg} / \mathrm{ml}$ a los 60 ' y en la mortalidad presentó su mayor efecto en $4 \mathrm{mg} / \mathrm{ml}$ a los 60' (34.88\%), mientras Martínez-Loredo et al. (2015) reportaron que los compuestos aislados del extracto etanólico de Heliopsis longipes causó una disminución total en la motilidad en $2 \mathrm{mg} / \mathrm{ml}$, una $\mathrm{CE}_{50}$ de $0.125 \mathrm{mg} / \mathrm{ml}$ a los 20 segundos y altos porcentajes de mortalidad llegando a un $98 \%$ en $0.5 \mathrm{mg} / \mathrm{ml}$ a $\operatorname{los} 30$ '.

Es interesante mencionar que obtuvimos metabolitos secundarios de naturaleza polar como los fenoles partir del extracto etanólico, siendo así excluidos los compuestos no polares como los terpenos, alcaloides indóli- 
cos, Caulerpina y Caulerpinina. Éstos últimos considerados los principales metabolitos del alga (Lemée et al., 1993), a los cuales se les atribuye la gran bioactividad negativa en eventos ligados a la reproducción de sus depredadores (Silvestre \& Tosti, 2010) (Pedrotti et al, 1996).

Si bien aún no se ha develado la composición completa de éste extracto, la alta cantidad de fenoles podrían ser los compuestos candidatos que afecten la motilidad y probablemente sea mediante la vía de depleción de ATP en las mitocondrias, ya que se ha visto este fenómeno en fenoles como Bisphenol-A en altas concentraciones, además incrementan la fosforilación de residuos en tirosina e inducen la proteína kinasa A responsable de la reacción acrosómica comprometiendo al espermatozoide a una falla en el proceso de fecundación (Rahman et al. 2015).

Estos resultados muestran una naturaleza contraceptiva promisoria ya que al no ser genotóxico ni citotóxico y gracias a sus características antiinflamatorias se presenta como una alternativa con ventajas sobre Nonoxynol-9 principal producto disponible en el mercado de origen sintético.

\section{CONCLUSIÓN}

El extracto etanólico de Caulerpa filiformis presenta una actividad espermostática a la concentración de $4 \mathrm{mg} / \mathrm{ml}$ a partir de los 15 minutos de exposición.

\section{REFERENCIAS BIBLIOGRÁFICAS}

1. Cengiz, S.; Cavas, L.; Yurdakoc, K. \& Aksu, S. (2012). Inhibition of xanthine oxidase by Caulerpenyne from Caulerpa prolifera. Turkish Journal of Biochemistry, 37(4): 445-451. doi:10.5505/ tjb.2012.98698

2. Da Matta, C.; De Souza, É.; De Queiroz, A.; De Lira, D.; De Araújo, M.; Cavalcante-Silva, L. \& Alexandre-Moreira, M. (2011). Antinociceptive and antiinflammatory activity from algae of the Genus Caulerpa. Marine Drugs, 9(3): 307-318. doi:10.3390/md9030307

3. Gandini, L., Lombardo, F., Paoli, D., Caponecchia, L., Familiari, G., Verlengia, C., Dondero, F., Lenzi, A., 2000. Study of apoptotic DNA fragmentation in human spermatozoa. Hum. Reprod., 15(4):830-839.

[doi:10.1093/humrep/15.4.830] 
4. Glombitza, K. \& Keusgen, M. 1995. Fuhalols and deshydroxyfuhalols from the brown alga Sargassum spinuligerum. Phytochemistry 38: 987-995.

5. Israel, A.; Einav, R. \& Seckbach, J. (2010). Seaweeds and their Role in Globally Changing Environments. Vasa, 15, 480. doi:10.1007/97890-481-8569-6

6. Jain, J.; Li, A.; Nucatola, D.; Minoo, P. \& Felix, J. (2005). Nonoxynol-9 induces apoptosis of endometrial explants by both caspasedependent and independent apoptotic pathways. Biol Reprod. 73(2): 382-388.

7. Jain J.; Li, A.; Minoo, P.; Nucatola, D. \& Felix, J. (2006). The effect of nonoxynol-9 on human endometrium. Contraception. 71(2):13742 .

8. Jain, A.; Kumar, L.; Kushwaha, B.; Sharma, M.; Pandey, A.; Verma, V.; Gupta, G. (2014). Combining a synthetic spermicide with a natural trichomonacide for safe, prophylactic contraception. Human Reproduction, 29(2): 242-252. doi:10.1093/humrep/det423

9. Lemée, R.; Pesando, D.; Durand-Clément, M.; Dubreuil, A.; Meinesz, A.; Guerriero, A. \& Pietra, F. (1993). Preliminary survey of toxicity of the green alga Caulerpa taxifolia introduced into the Mediterranean. Journal of Applied Phycology, 5(5): 485-493. doi:10.1007/ BF02182507

10. Martinez-Loredo, E.; Izquierdo-Vega, J.; Cariño-Cortes, R.; CiliaLópez, V.; Madrigal-Santillán, E.; Zuñiga-Pérez, C. \& Sánchez-Gutiérrez, M. (2015).

Effects of Heliopsis longipes ethanolic extract on mouse spermatozoa in vitro. Pharmaceutical Biology, 1-6. doi:10.3109/13880209.20 15.1033560

11. Mayer, A.; \& Hamann, M. (2002). Marine pharmacology in 1999: Compounds with antibacterial, anticoagulant, antifungal, anthelmintic, anti-inflammatory, antiplatelet, antiprotozoal and antiviral activities affecting the cardiovascular, endocrine, immune and nervous systems, and other misc. Comparative Biochemistry and Physiology - $C$ Toxicology and Pharmacology. doi:10.1016/S1532-0456(02)000947 
12. Nicoletti, E.; Della Pieta', F.; Calderone, V.; Bandecchi, P.; Pistello, M.; Morelli, I. \& Cinelli, F. (1999). Antiviral properties of a crude extract from a green alga Caulerpa taxifolia (Vahl) C. Agardh. Phytotherapy Research, 13(3): 245-247. doi:10.1002/(SICI)10991573(199905)13:3<245::AID-PTR424>3.0.CO;2-P

13. Nithya, R.; Anuja, M.; Rajamanickam, C. \& Indira, M. (2012). Rat sperm immobilisation effects of a protein from Ricinus communis (Linn.): an in vitro comparative study with nonoxynol-9. Andrologia, 1-7. doi:10.1111/j.1439-0272.2012.01291.x

14. Ohta, E.; Uy, M.; Ohta, S.; Yanai, M.; Hirata, T. \& Ikegami, S. (2008). Antifertilization activity of a spirocyclic sesquiterpene isocyanide isolated from the marine sponge Geodia exigua and related compounds. Bioscience, Biotechnology, and Biochemistry, 72(7): 1764-1771. doi:10.1271/bbb.80071

15. Ospina, L.; Alvarez, A.; Arango, V.; Cadavid, A. \& CARDONA, W. (2013). Actividad espermicida y citotoxica del extracto de Sapinus saponaria L. (jaboncillo). Revista Cubana de Plantas Medicinales Vol. 18(2): 187-200.

16. Paul, D., Bera, S., Jana, D., Maiti, R., \& Ghosh, D. (2006). In vitro determination of the contraceptive spermicidal activity of a composite extract of Achyranthes aspera and Stephania hernandifolia on human sperm. Contraception 73(3), 284-288. doi:10.1016/j.contraception.2005.07.014

17. Pedrotti, M. L., Marchi, B., \& Lemee, R. (1996). Effects of Caulerpa taxifolia secondary metabolites on the embryogenesis, larval development and metamorphosis of the sea urchin Paracentrotus lividus. Oceanologica Acta, 19: 255-262.

18. Prakash, S., Ravikumar, S., Reddy, K. V. R., \& Kannapiran, E. (2013). Spermicidal activity of Indian seaweeds: An in vitro study. Andrologia, 46(4): 408-416. doi:10.1111/and.12096

19. Qiu, Y.; Wang, L.; Jia, Y.; Yang, D.; Zhang, M.; Zhang, Y. \& Gai, L. (2011). Effects of the crude extract of Polygala tenuifolia Willd on human sperm in vitro . Journal of Zhejiang University. Science. B, 12(6): 448-454. doi:10.1631/jzus.B1000347 
20. Rahman, M.; Kwon, W.; Lee, J.; Yoon, S.; Ryu, B. \& Pang, M. (2015). Bisphenol-A Affects Male Fertility via Fertility-related Proteins in Spermatozoa. Scientific Reports, 5(1), 9169. doi:10.1038/srep09169

21. Richter, P.; Schubert, G.; Schaible, A.; Cavas, L.; Werz, O. \& Pohnert, G. (2014). Caulerpenyne and Related Bis-enol Esters Are Novel-Type Inhibitors of Human 5-Lipoxygenase. ChemMedChem, n/a-n/a. doi:10.1002/cmdc.201402065

22. Rovirosa, J.; Soto, H.; Cueto, M.; Dárias, J.; Herrera, J. \& San-Martín, A. (1999). Sesquiterpenes from Laurencia claviformis. Phytochemistry, 50(5): 745- 748. doi:10.1016/S0031-9422(98)00617-7

23. Silvestre, F. \& Tosti, E. (2010). Impact of marine drugs on cytoskeletonmediated reproductive events. Marine Drugs, 8(4): 881-915. doi:10.3390/md8040881

24. Smyrniotopoulos, V.; Abatis, D.; Tziveleka, L.; Tsitsimpikou, C.; Roussis, V.; Loukis, A. \& Vagias, C. (2003). Acetylene sesquiterpenoid esters from the green alga Caulerpa prolifera. Journal of Natural Products, 66(1): 21-24. doi:10.1021/np0202529

25. Suhaiman, L.; Carlos de-Rosas, J.; Sartor, T.; Palmada, N.; Giordano, O. \& Lopez, L. (2011). Effect of dehydroleucodine on the reproductive tract of male mice. Andrologia, 43(5): 297-302. doi:10.1111/ j.1439-0272.2010.01053.x

26. WHO. (2010). Examination and processing of human semen (Fifth Edit). Switzerland. 